\title{
4. Der Übergang in den Ruhestand: Alter, Pfade und Ausstiegspläne
}

\author{
Heribert Engstler \& Laura Romeu Gordo
}

\section{Kernaussagen}

Trotz eines längeren Verbleibs im Erwerbsleben arbeiten immer weniger ältere Erwerbstätige bis zum Beginn ihrer Altersrente: Der Anteil der 66- bis 71-Jährigen, die bis zum Renteneintritt erwerbstätig waren, ist zwischen 1996 und 2014 von 62,0 auf 46,6 Prozent gesunken. Zugenommen hat dagegen der Anteil der Personen, die vor dem Rentenbeginn arbeitslos waren oder in der Freistellungsphase der Altersteilzeit.

Die Ost-West-Unterschiede in den Übergangspfaden haben sich bei Männern verringert, bei Frauen nicht: Bei ostdeutschen Frauen hat Arbeitslosigkeit vor dem Rentenbeginn stärker zugenommen (1996: 1,0 Prozent; 2014: 39,7 Prozent) als bei westdeutschen Frauen (2014: 13,4 Prozent). Westdeutsche Frauen sind vor Beginn der Altersrente immer noch häufiger Hausfrauen (27,2 Prozent) als ostdeutsche Frauen (2014: 0,8 Prozent).

Nach Abschaffung der staatlichen Förderung sinkt die Inanspruchnahme von Altersteilzeit: Im Jahr 2014 befinden sich nur 6,9 Prozent der 55- bis 65-jährigen abhängig Beschäftigten in der Altersteilzeit (2008: 16,9 Prozent). Auch planen weniger Beschäftigte, diese in Anspruch zu nehmen. Altersteilzeit wird überdurchschnittlich oft von qualifizierten Beschäftigten in großen Industriebetrieben und meist im Blockmodell für einen frühzeitigen Ausstieg genutzt.

Immer mehr Erwerbstätige planen, bis zum Alter von 65 Jahren oder länger zu arbeiten: Von den 40- bis 59-Jährigen des Jahres 2014 planen 39,5 Prozent, mit 65 Jahren oder später mit der Erwerbstätigkeit aufzuhören (1996: 18,2 Prozent), nur noch 15,9 Prozent mit 60 Jahren oder früher. Einen späten Ausstieg planen insbesondere Hochgebildete, Selbstständige und Angestellte mit Vorgesetztenfunktion.

\subsection{Einleitung}

In der Diskussion über die Bewältigung der Auswirkungen des demografischen Wandels auf den Arbeitsmarkt und die Alterssicherung wird der Verlängerung des Arbeitslebens und der Flexibilisierung des Übergangs in den Ruhestand eine hohe Bedeutung zugeschrieben. Besonderen politischen Ausdruck fand dies in den Rentenreformen zur schrittweisen Anhebung des Anspruchsalters beziehungsweise der weitgehenden Abschaffung vorgezogener Altersrenten und der Anhebung der Regelaltersgrenze. Allerdings wurden entgegen dieser allgemeinen Ausrichtung mit der vorzeitigen abschlagfreien Rente für besonders langjährig Versicherte nach 45 Versicherungsjahren auch Regelungen eingeführt, die dem Ziel des längeren Verbleibs im Erwerbsleben zuwider laufen. Kritiker der Altersgrenzenanhebungen befürchten, dass ein wachsender Teil älterer Arbeitskräfte aus gesundheitlichen und Arbeitsmarktgründen nicht bis zum Erreichen der 
Regelaltersgrenze arbeiten kann und daher die Zeit bis zum Rentenbeginn überbrücken muss oder vorzeitig mit Abschlägen in den Rentenbezug geht. Andererseits wächst auch die Kritik am unflexiblen automatischen Ende des Arbeitsverhältnisses mit Erreichen der Regelaltersgrenze. Die Bundesregierung hat sich daher zum Ziel gesetzt, die Rahmenbedingungen für flexiblere Übergänge in den Ruhestand zu verbessern (CDU, CSU, \& SPD 2013: 52).

Die Förderung der Altersteilzeit sollte einen längeren Verbleib im Erwerbsleben unterstützen und einen gleitenden Übergang in den Ruhestand ermöglichen. Die Altersteilzeit ist eine besondere Form der Teilzeitbeschäftigung in den Jahren vor dem Renteneintritt. Der Arbeitnehmer oder die Arbeitnehmerin vereinbaren mit dem Arbeitgeber meist gestützt auf tarifvertragliche Regelungen oder Betriebsvereinbarungen - entweder eine gleichmäßige Reduzierung der wöchentlichen Arbeitszeit bis zum Rentenbeginn (Gleichverteilungsmodell) oder die hälftige Aufteilung der verbleibenden Arbeitsjahre in eine Arbeitsphase mit ungekürzter Wochenarbeitszeit und die anschließende Phase der Freistellung von der Arbeitsleistung (Blockmodell). Wesentliches Merkmal der Altersteilzeit ist die Aufstockung des Regelarbeitsentgelts und der Beiträge zur Rentenversicherung durch den Arbeitgeber. Nach einer Reform des Altersteilzeitgesetzes im Jahr 1996 förderte die Bundesagentur für Arbeit unter bestimmten Voraussetzungen (Ersatzeinstellung von Arbeitslosen, Übernahme von Ausbildungsabsolventen) Altersteilzeitarbeitsplätze durch eine weitgehende Kompensation des Mehraufwands. Allerdings wurde diese Regelung wieder abgeschafft und die staatliche Förderung der Altersteilzeit läuft aus. Sie betrifft nur noch Fälle, in denen die Altersteilzeit vor dem 01.01.2010 angetreten wurde und die Beschäftigten zu diesem Zeitpunkt ihr 55. Lebensjahr vollendet hatten. Der Wegfall der staatlichen Förderung hat es für Unternehmen finanziell weniger attraktiv werden lassen, ihren älteren Beschäftigten die Altersteilzeit anzubieten, wenn auch nach Schätzungen von Brussig, Knuth, and Wojtkowski (2009) nur für weniger als die Hälfte der Altersteilzeitfälle eine staatliche Förderung in Anspruch genommen wurde. Aber viele Tarifverträge und Betriebsvereinbarungen zur Altersteilzeit waren an die Option der staatlichen För- derung gebunden (ebd.). Zudem wurde die Möglichkeit der vorgezogenen gesetzlichen Altersrente nach Altersteilzeit für alle nach 1951 Geborenen abgeschafft. Für diese gelten daher wieder die normalen Voraussetzungen des Rentenübergangs. Andererseits gibt es immer noch viele Altersteilzeitregelungen in den Tarifverträgen (Fröhler, Fehmel, \& Klammer 2013: 690ff). Daher stellt sich die Frage, ob die veränderten Rahmenbedingungen in den letzten Jahren zu einem Rückgang der Inanspruchnahme von Altersteilzeit und Veränderungen der Modelle geführt haben. Bislang wurde die Altersteilzeit hauptsächlich als Blockmodell und damit faktisch als Vorruhestandslösung genutzt (Brussig et al. 2009; Wanger 2009; Naumann \& Romeu Gordo 2010).

Vor diesem Hintergrund widmet sich der vorliegende Beitrag zunächst der Entwicklung des Alters und der Pfade des Übergangs in den Ruhestand bis zu den Geburtsjahrgängen, die erst vor kurzem in den Ruhestand eingetreten sind. Im Vordergrund steht dabei die Frage, ob der Aufschub des Rentenbeginns auch mit einem entsprechend längeren Verbleib in der Erwerbstätigkeit einhergeht. Hält der Anstieg des Alters, in dem die berufliche Tätigkeit beendet wird, Schritt mit dem steigenden Renteneintrittsalter oder verlängert sich die Wartezeit zwischen Erwerbsausstieg und Rentenbeginn?

Vermutet wird, dass sich in der Kohortenabfolge zwar beide Ereignisse in ein höheres Alter verlagert haben, aber das Renteneintrittsalter schneller gestiegen ist als das Erwerbsaustrittsalter. Dies müsste sich auch in den Übergangspfaden zeigen, die mit der Erwartung betrachtet werden, dass immer weniger ältere Arbeitskräfte nahtlos aus der Erwerbstätigkeit in den Rentenbezug übergehen. Aufgrund der unterschiedlichen Arbeitsmarktentwicklungen und Erwerbsbeteiligungsmuster zwischen Ostund Westdeutschland und zwischen Männern und Frauen werden im Hinblick auf das Alter und die Pfade des Übergangs in den Ruhestand auch Ost-West- und geschlechtsspezifische Unterschiede erwartet. Für Ostdeutschland erwarten wir relativ mehr Übergänge aus der Arbeitslosigkeit. Für Frauen erwarten wir häufiger als für Männer einen Rentenbeginn nach längerer erwerbsfreier Haushaltstätigkeit. Aufgrund der 
steigenden Erwerbsbeteiligung westdeutscher Frauen (Simonson, Romeu Gordo, \& Titova 2011) nehmen wir allerdings an, dass der Rentenzugang aus vorheriger Hausfrauentätigkeit in der Kohortenabfolge seltener geworden ist. Zusätzlich werden bildungsspezifische Unterschiede betrachtet, da wir davon ausgehen, dass es höhergebildeten Personen leichter möglich ist, bis direkt zum Rentenbeginn im Arbeitsprozess zu bleiben. Von der Einführung der Altersrente für besonders langjährig Versicherte erwarten wir für unsere Analysen zum erfolgten Übergang in den Ruhestand noch keine Auswirkungen, da die von uns betrachteten Geburtskohorten aufgrund ihres Alters nicht mehr in den Genuss der zum Juli 2014 eingeführten ,Rente mit $63^{`}$ gekommen sind.

Anschließend wird untersucht, ob das Auslaufen der staatlichen Förderung der Altersteilzeit einhergeht mit einem Rückgang der realisierten und geplanten Inanspruchnahme der Altersteilzeit bei älteren Beschäftigten. Geprüft wird auch, ob die Dominanz des Blockmodells nachlässt oder ob der Altersteilzeit ungebrochen die Funktion eines frühzeitigen Ausscheidens aus der aktiven Arbeit zukommt.

Um Hinweise auf die zukünftige Entwicklung zu erhalten, werden schließlich noch die Übergangspläne der im Erwerbsleben stehenden älteren Arbeitskräfte betrachtet, zu denen aktuell bereits die geburtenstarken Jahrgänge

\subsection{Datengrundlage ${ }^{1}$}

Daten. Die Untersuchung des Übergangsalters und der Pfade in den Ruhestand stützt sich auf die Retrospektivangaben der jeweils 66- bis 71-jährigen Befragten der Basisstichproben 1996, 2002, 2008 und 2014 des Deutschen Alterssurveys (DEAS). Einbezogen sind alle Personen dieses Alters knapp oberhalb der Regelaltersgrenze, die an-

1 Die Daten des DEAS können für wissenschaftliche Zwecke kostenlos beim Forschungsdatenzentrum des DZA (www.fdz-dza.de) bezogen werden. zählen. Die Untersuchung des geplanten Ausstiegsalters gibt Hinweise, inwieweit die Betroffenen sich darauf einstellen, länger erwerbstätig $\mathrm{zu}$ bleiben. In einer früheren Studie mit Daten des Deutschen Alterssurveys konnte gezeigt werden, dass die Ausstiegspläne ein guter Indikator für das spätere tatsächliche Ausstiegsverhalten sind (Engstler 2006).

Zusammenfassend werden die folgenden Fragen untersucht:

1. Steigt das Renteneintrittsalter schneller als das Berufsaustrittsalter und nehmen die direkten Übergänge aus der Erwerbstätigkeit in den Ruhestand $a b$ ? Gilt dies für Männer und Frauen, für Ost- und Westdeutsche und für die unterschiedlichen Bildungsgruppen gleichermaßen?

2. Verringert sich nach Abschaffung der staatlichen Förderung der Anteil derer, die vor dem Wechsel in den Ruhestand eine Altersteilzeitbeschäftigung planen oder ausüben? Wer hat geringere Chancen auf die Inanspruchnahme von Altersteilzeit?

3. Haben die Vorstellungen von einem frühen Ruhestand ausgedient? Planen die geburtenstarken Jahrgänge, bis zum Alter 65 oder länger zu arbeiten? Unterscheiden sich die Ausstiegspläne nach dem Qualifikations- und Belastungsniveau der Erwerbstätigen?

geben, eine Altersrente oder Pension aus eigener Erwerbstätigkeit zu beziehen. Die Auswahl dieser Sechs-Jahres-Altersgruppe ermöglicht - passend zum Sechs-Jahres-Abstand der Ziehung neuer DEAS-Basisstichproben - einen trennscharfen Kohortenvergleich des Übergangsverhaltens der vier Geburtsjahrgangsgruppen 1925/30, 1931/36, $1937 / 42$ und 1943/48. Auswahlgrundlage für die Untersuchung des Wandels des geplanten Ausstiegsalters aus der Erwerbstätigkeit sind alle noch nicht im Ruhestand befindlichen Erwerbstätigen 
unter 60 Jahren aller vier Basisstichproben. Die Beschränkung auf die unter 60-Jährigen erfolgt, um allen in die Analyse Einbezogenen gleichermaßen eine Nennungsbreite von 60 und mehr Jahren für das geplante Ausstiegsalter zu ermöglichen. Die Darstellung zur Entwicklung der ausgeübten oder beabsichtigen Altersteilzeitbeschäftigung beruht auf den Auskünften aller abhängigen Beschäftigten im vollendeten Alter von 55 bis 65 Jahren, da dies die üblichen Unter- und Obergrenzen der Altersteilzeitbeschäftigung sind. Alle Variablen beruhen auf Angaben des persönlichen mündlichen Interviews.

Realisiertes Erwerbsausstiegs- und Renteneintrittsalter. Betrachtet wird das Ausstiegsalter aus der hauptberuflichen Erwerbstätigkeit (ungeachtet einer möglichen Erwerbstätigkeit im Ruhestand) und das Alter beim Beginn des Bezugs der Altersrente oder Pension der 66- bis 71-Jährigen.

Übergangspfade. Die Pfade in den Ruhestand werden anhand der Angaben der Befragten zum Status unmittelbar vor dem Beginn der Altersrente oder Pension unterschieden. Es werden sechs Kategorien gebildet: (1) Übergang aus aktiver Erwerbstätigkeit, das heißt die Person hat bis zum Rentenbeginn gearbeitet, (2) aus der Freistellungsphase der Altersteilzeit, (3) aus der Arbeitslosigkeit, (4) aus dem Vorruhestand, (5) aus der Erwerbsunfähigkeit oder längerer Krankheit (mit Krankengeldbezug), (6) aus der Haushaltstätigkeit oder sonstigem Status.

Geplantes Ausstiegsalter. Der Indikator beruht auf den Antworten der Erwerbstätigen auf die Frage, mit welchem Alter sie planen, ihre Erwerbstätigkeit zu beenden. Es kann ein konkretes Altersjahr genannt werden oder die Angabe ,weiß noch nicht. Die Altersangaben werden zu den vier Kategorien ,mit 60 Jahren oder früher', ,mit 61-64 Jahren', ,mit 65 Jahren' und ,mit 66 Jahren oder später' zusammengefasst, die Angabe ,weiß noch nicht' bildet eine zusätzliche Kategorie.

Altersteilzeit. Die Darstellung zur Entwicklung der ausgeübten oder beabsichtigen Altersteilzeitbeschäftigung beruht auf den Auskünften aller abhängigen Beschäftigten im vollendeten Alter von 55 bis 65 Jahren. Da erst ab der Erhebung des Jahres 2002 Fragen zur Altersteilzeit im DEAS enthalten sind, kann die Entwicklung von 2002 bis 2014 untersucht werden.

Gruppierungsvariablen. Es werden Geschlechtsunterschiede (männlich/weiblich) und regionale Unterschiede zwischen West- und Ostdeutschland untersucht. Westdeutschland umfasst die alten Bundesländer einschließlich dem früheren Westteil Berlins, Ostdeutschland die neuen Bundesländer inklusive dem früheren Ostteil Berlins. Zur Untersuchung von Bildungsunterschieden werden - basierend auf einer reduzierten ISCED-Klassifikation - drei Bildungsgruppen verglichen: Personen mit niedrigem, mittlerem und hohem Bildungsniveau (vgl. Kapitel 2). Bei den Darstellungen zur Altersteilzeit und/oder dem geplanten Ausstiegsalter werden bivariat auch Unterschiede hinsichtlich folgender Aspekte berücksichtigt: Betriebsgröße (gemessen an der Beschäftigtenzahl: unter 20, 20 bis unter 200, 200 bis unter 2000, 2000 und mehr), Wirtschaftszweig des Betriebs (Landwirtschaft/ Handwerk, Industrie, Handel/Dienstleistung, Öffentlicher Dienst) und berufliche Stellung der Beschäftigten (un-/angelernte Arbeiter, Facharbeiter/Meister, einfache/qualifizierte Angestellte, hochqualifizierte/vorgesetzte Angestellte, Beamte, Selbstständige (inkl. mithelfende Angehörige)). Untersucht wird auch, welchen Einfluss die empfundene Arbeitsbelastung auf die Ausstiegspläne hat. Verglichen werden hierfür Erwerbstätige, die sich in mindestens einem von vier Belastungsbereichen ziemlich oder sehr belastet fühlen (auf einer fünf-stufigen Skala), mit Erwerbstätigen, die sich in keinem Bereich ziemlich oder sehr belastet fühlen. Die Bereiche sind: anstrengende oder einseitige körperliche Aktivitäten, erschwerte Umweltbedingungen am Arbeitsplatz (Lärm, Hitze, Staub etc.), Stress, viele neue Anforderungen.

Analyse. Im Text und in den Abbildungen werden jahres- und gruppenspezifische gewichtete Mittelwerte oder prozentuale Verteilungen angegeben. Um Unterschiede zwischen Gruppen und/ oder Jahren auf statistische Signifikanz $(p<, 05) \mathrm{zu}$ testen, wurden in Abhängigkeit vom Skalenniveau logistische Regressionen oder Kovarianzanalysen berechnet, wobei für die Stratifizierungsvariablen Altersgruppe, Geschlecht und Region (Ost-/Westdeutschland) kontrolliert wurde. 


\subsection{Entwicklung des Übergangsalters und der Pfade in den Ruhestand}

Wie Abbildung 4-1 zeigt, ist das durchschnittliche Erwerbsaustrittsalter noch bis Ende des vergangenen Jahrzehnts gesunken, während das Renteneintrittsalter schon zu Beginn der 2000er Jahre seine Talsohle erreicht hatte. Die 66- bis 71-Jährigen des Jahres 2008 sind mit durchschnittlich 56,5 Jahren aus dem Erwerbsleben ausgeschieden und mit 61,4 Jahren in Rente gegangen. Im Vergleich zu 1996 bedeutete dies eine Absenkung des Erwerbsaustrittsalters um 1,2 Jahre, das des Rentenbeginns um 0,7 Jahre. In den sechs Jahren nach 2008 verlagerte sich dann sowohl der Berufsausstieg als auch der Renteneintritt in ein höheres Alter. Die 66- bis 71-Jährigen des Jahres 2014 (Geburtsjahrgänge 1943/48) gingen mit durchschnittlich 62,3 Jahren in Rente, nachdem sie ihre Berufstätigkeit im Mittel mit 57,8 Jahren beendet haben. Entgegen unserer Erwartung verringerte sich die durchschnittliche Dauer zwischen Erwerbsende und Rentenbeginn nach 2008 wieder, weil das Ausstiegsalter etwas stärker gestiegen ist als das Renteneintrittsalter.

\section{Abbildung 4-1: Durchschnittliches Erwerbsaustritts- und Renteneintrittsalter der 66- bis 71-Jährigen mit} Rentenbezug, in den Jahren 1996, 2002, 2008 und 2014 (in Jahren)

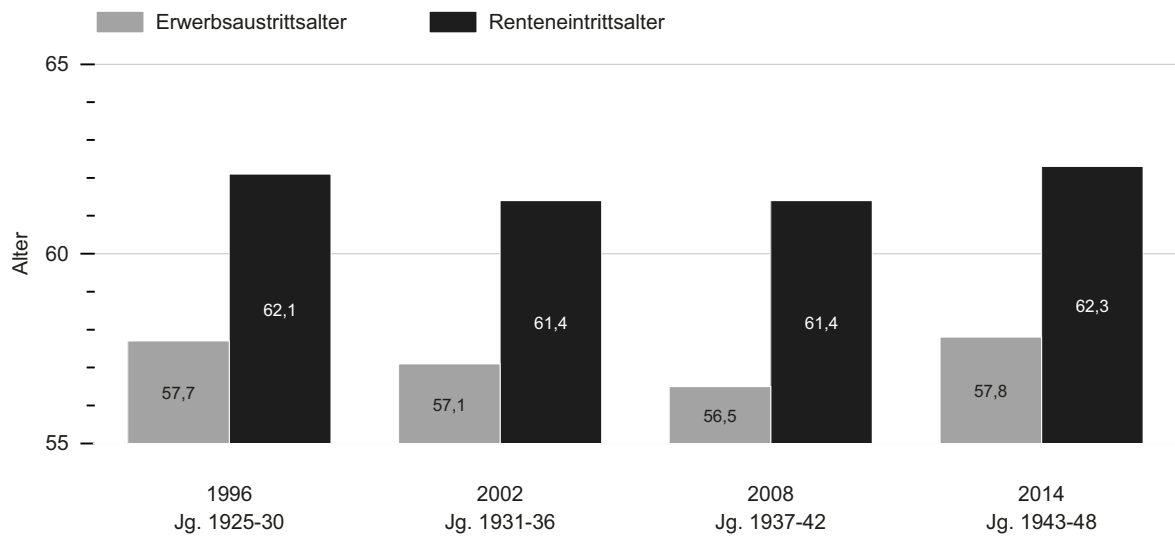

Quelle: DEAS 1996 ( $n$ = 521 für Erwerbsaustrittsalter; 509 für Renteneintrittsalter), 2002 ( $n=346$ für Erwerbsaustrittsalter; 347 für Renteneintrittsalter), 2008 ( $n=1.058$ für Erwerbsaustrittsalter; 1.060 für Renteneintrittsalter), 2014 ( $n=827$ für Erwerbsaustrittsalter; 830 für Renteneintrittsalter), gewichtet, gerundete Angaben; $(p<, 05)$. Altersrückgänge zwischen 1996 und 2008 und Altersanstiege zwischen 2008 und 2014 sind signifikant.

Hinter dieser durchschnittlichen Entwicklung stehen unterschiedliche Veränderungen der nachberuflichen Dauer bis zum Renteneintritt bei den einzelnen Übergangspfaden in den Ruhestand. Bei denjenigen, die als Hausfrau/ -mann oder aus anderen Gründen bereits lange vor dem Rentenbeginn aus dem Arbeitsleben ausgeschieden sind, hat sich die Nichterwerbs- phase vor der Rente verkürzt. Bei Personen, die vor dem Renteneintritt arbeitslos wurden oder im Vorruhestand waren, hat sich die Dauer zwischen Erwerbsausstieg und Rentenbeginn hingegen - wie erwartet - verlängert. Im Durchschnitt scheiden diese nun bereits 4,5 Jahre vor dem Renteneintritt aus dem Erwerbsleben aus (Abbildung 4-2). 


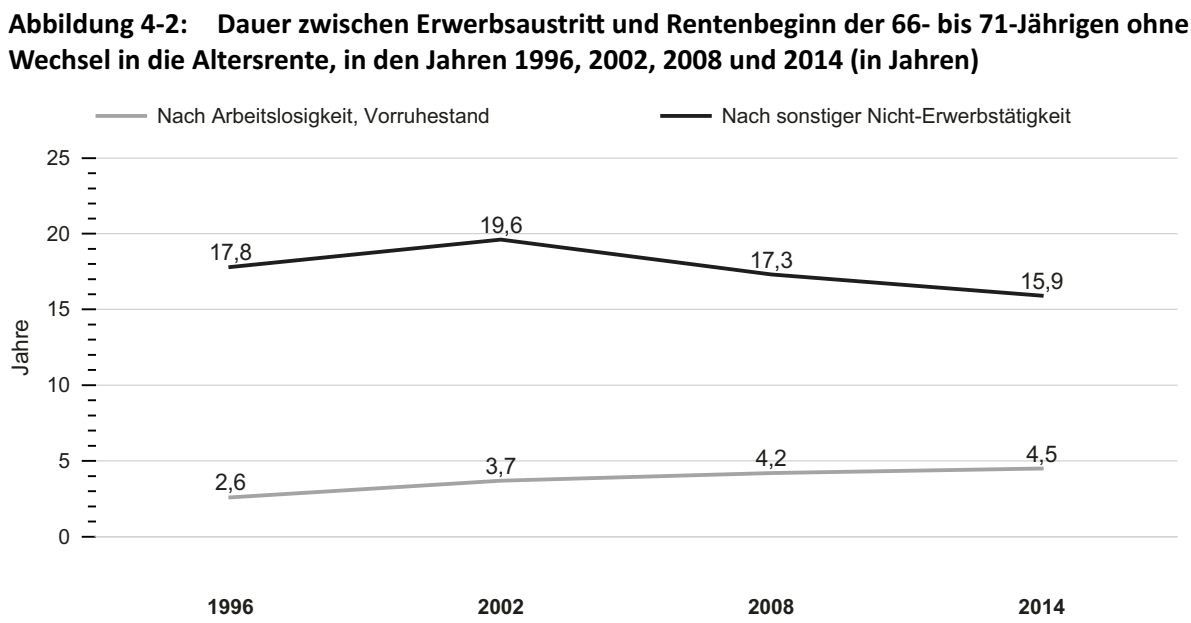

$\overline{\text { Quelle: DEAS } 1996}(n=168), 2002(n=128), 2008(n=429), 2014(n=311)$, gewichtet, gerundete Angaben; $(p<, 05)$. Signifikante Zunahme der Dauer bis Rentenbeginn nach Arbeitslosigkeit, Vorruhestand zwischen 1996 und 2014. Signifikanter Rückgang der Dauer bis Rentenbeginn nach sonstiger Nicht-Erwerbstätigkeit zwischen 2002 und 2014.

\section{Trotz eines längeren Verbleibs im Erwerbsleben arbeiten immer weniger ältere Erwerbstätige bis zum Beginn ihrer Altersrente.}

Zudem wächst der Anteil derer, die vor dem Ruhestand arbeitslos werden. Zwischen 1996 und 2014 hat sich dieser Übergangspfad auf 15,5 Prozent vervierfacht (Abbildung 4-3). Die Entlassung in die Arbeitslosigkeit am Ende des Erwerbslebens löst damit teilweise die Ausgliederung über Vorruhestandsregelungen ab. Über den Vorruhestand wechseln im Jahr 2014 nur noch 3,0 Prozent in die Altersrente, 1996 waren es noch 10,1 Prozent gewesen. Neben der Arbeitslosigkeit hat auch der vorzeitige Ausstieg über die geblockte Altersteilzeit an Bedeutung gewonnen. Von den 66- bis 71-Jährigen des Jahres 2014 sind 15,0 Prozent aus der
Freistellungsphase in die Altersrente gewechselt, 2002 waren es erst 0,6 Prozent gewesen. ${ }^{2}$ Es ist aber unwahrscheinlich, dass sich dieser Aufwärtstrend der Altersteilzeit nach der erfolgten Abschaffung der staatlichen Förderung fortsetzen wird (siehe hierzu Abschnitt 4.4). Stagnierend bis rückläufig sind die Anteile der Personen, die vor Beginn der Altersrente Hausfrau oder Hausmann, länger krank oder erwerbsunfähig oder aus anderen Gründen nicht erwerbstätig sind. Insgesamt zeigt sich für den untersuchten Zeitraum eine sehr klare Entwicklung: Immer weniger Menschen sind bis zum Rentenbeginn aktiv erwerbstätig geblieben. Waren von den 66- bis 71-Jährigen des Jahres 1996 noch 62,0 Prozent bis unmittelbar vor dem Renteneintritt erwerbstätig gewesen, trifft dies im Jahr 2014 nur noch auf 46,6 Prozent dieser Altersgruppe zu.

2 Bei den im Ruhestand Befindlichen des Jahres 1996 wurde der Übergang aus Altersteilzeit wegen fehlender Verfügbarkeit nicht erhoben. 
Abbildung 4-3: Situation der 66- bis 71-Jährigen vor Beginn der Altersrente, in den Jahren 1996, 2002, 2008 und 2014 (in Prozent)
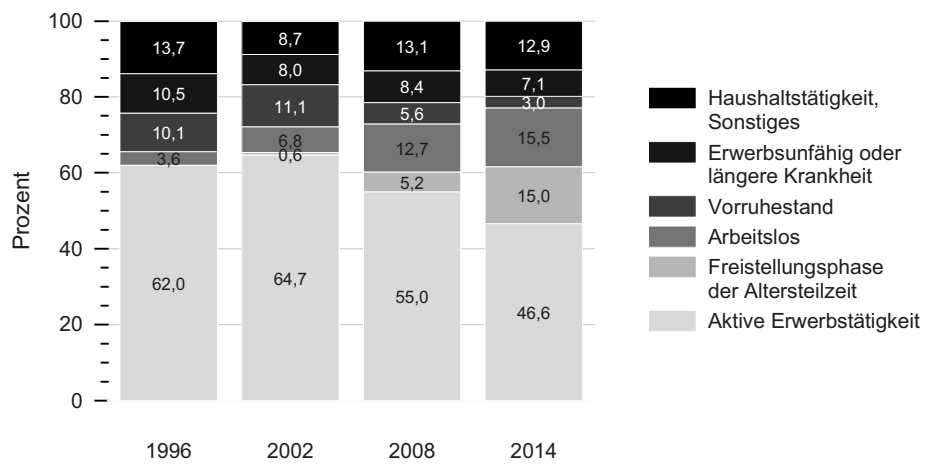

Quelle: DEAS $1996(n=527), 2002(n=347), 2008(n=1.060), 2014(n=831)$, gewichtet, gerundete Angaben; $(p<, 05)$. Signifikante Veränderungen in allen Kategorien außer Haushaltstätigkeit, Sonstiges zwischen 1996 und 2014.

Hinter dieser Gesamtveränderung verbergen sich unterschiedliche Entwicklungen in den alten und neuen Bundesländern und zwischen den Geschlechtern. Einen besonders starken Einbruch beim direkten Rentenübergang aus der Erwerbstätigkeit erlebten ostdeutsche Frauen: Von den 66- bis 71-Jährigen des Jahres 1996 hatten noch 76,7 Prozent bis direkt zum Rentenbeginn gearbeitet, im Jahr 2014 nur noch 36,7 Prozent (Abbildung 4-4). Vom Spitzenreiter wurden sie damit zum Schlusslicht. Im Gegenzug hat bei ihnen der Renteneintritt aus der Arbeitslosigkeit in diesen 18 Jahren von 1,0 auf 39,7 Prozent zugenommen. Bei den Männern in den neuen Bundesländern verlief die Entwicklung positiver. Zwar waren auch sie anfangs von der starken Zunahme der Arbeitslosigkeit und des Vorruhestands am Ende des Erwerbslebens betroffen, nach 2002 gelang es ihnen aber wieder in steigendem Maße, bis unmittelbar vor dem Rentenzugang erwerbstätig zu bleiben (vgl. Tabelle A 4-3 im Anhang). Zuletzt überholten sie damit sogar die westdeutschen Männer, wenn auch in erster Linie durch ihre geringere Inanspruchnahme der Altersteilzeit (Abbildung 4-4).

\section{Die Ost-West-Unterschiede bei den Übergangspfaden haben sich nur bei Männern verringert, bei Frauen nicht.}

Die geblockte Altersteilzeit wird am häufigsten von westdeutschen Männern für den vorzeitigen Abschied aus der aktiven Erwerbstätigkeit genutzt. Entgegen unseren Erwartungen zeigt sich bei den Frauen in den alten Bundesländern keine kontinuierliche Zunahme des Arbeitens bis zum Rentenbeginn. Zwar erhöhte sich bei ihnen zunächst zwischen 1996 und 2002 der Anteil direkter Rentenübergänge aus der Erwerbstätigkeit, seither hat er sich jedoch wieder unter das Ausgangsniveau verringert (1996: 55,8 Prozent; 2014: 41,5 Prozent). Anteilig häufiger geworden sind bei westdeutschen Frauen in erster Linie die Rentenübergänge nach vorheriger Arbeitslosigkeit, Vorruhestand und geblockter Altersteilzeit. Und trotz Rückgangs gelangt immer noch mehr als ein Viertel der westdeutschen Frauen als Hausfrau oder sonstige Nichterwerbsperson in die Ruhestandsphase. 


\section{Abbildung 4-4: Situation der 66- bis 71-Jährigen vor Beginn der Altersrente, nach Geschlecht und Landesteil, in den Jahren 1996 und 2014 (in Prozent)}

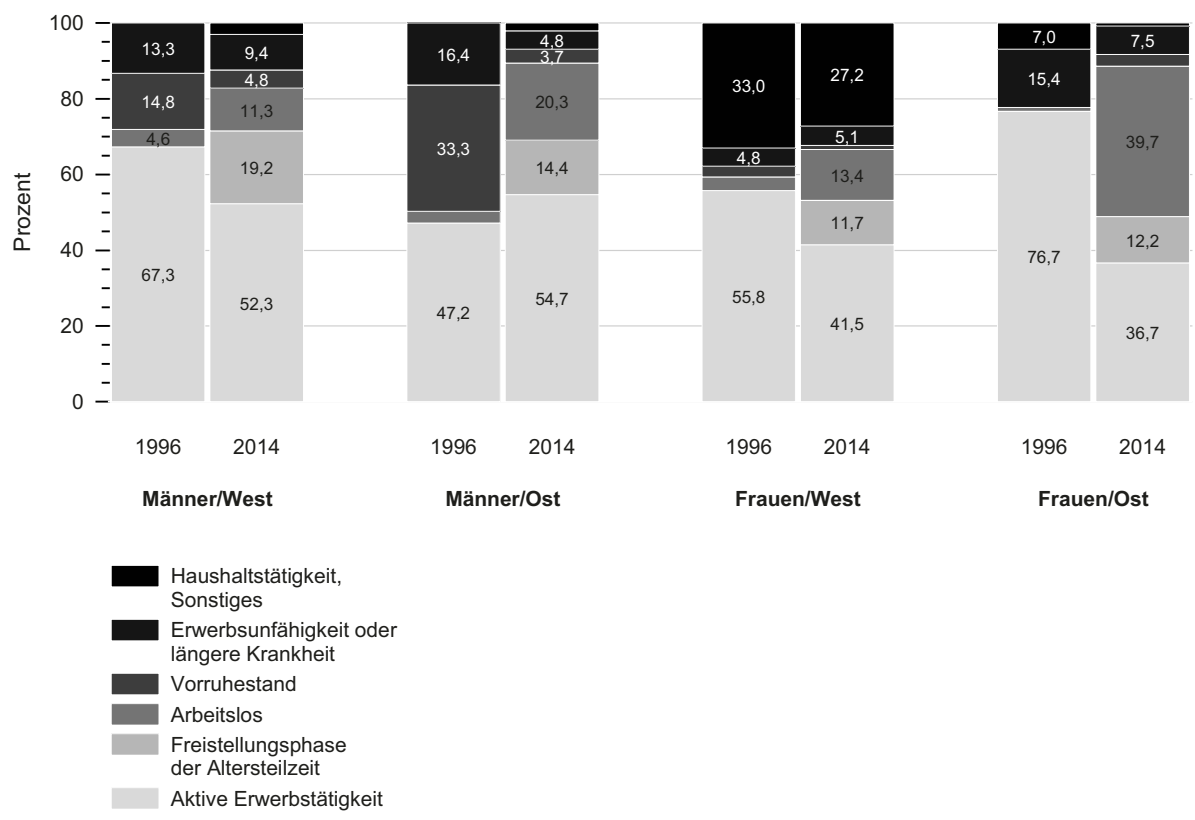

Quelle: DEAS 1996 ( $n=527), 2014(n=831)$, gewichtet, gerundete Angaben; $(p<, 05)$.

Für alle Kategorien signifikante Unterschiede zwischen 1996 und 2014. Signifikante Regions- und Geschlechtsunterschiede für alle Kategorien in den Jahren 1996 und 2014 .

Ob Menschen bis zum Rentenbeginn erwerbstätig bleiben und ein Arbeitsverhältnis aufrechterhalten, hängt auch von ihrem Bildungsniveau ab. Dies zeigt sich deutlich an den Übergangspfaden in den Ruhestand der 66- bis 71-Jährigen des Jahres 2014. Je höher ihr Bildungsniveau ist, desto häufiger sind sie bis zum Rentenbeginn aktiv erwerbstätig geblieben oder konnten aus der gut abgesicherten Position der Altersteilzeit in den Ruhestand wechseln (Abbildung 4-5). Niedriggebildete (Personen ohne abgeschlossene Berufsausbildung) haben ein erhöhtes Risiko, bereits vor dem Wechsel in die Altersrente aufgrund von Arbeitslosigkeit, Krankheit oder Erwerbsunfähigkeit aus dem Erwerbsprozess auszuscheiden oder nach der Familienphase keine dauerhafte Rückkehr in die Berufstätigkeit zu schaffen. Letzteres betrifft vor allem Frauen. Insgesamt zeigt sich, dass das steigende Erwerbsausstiegs- und Renteneintrittsalter begleitet wird von einem abnehmenden Anteil derer, die bis zum Rentenbeginn aktiv erwerbstätig bleiben, und dass sich die Übergangspfade in den Ruhestand immer noch zwischen den alten und neuen Bundesländern unterscheiden und soziale Unterschiede aufweisen. Die Arbeitslosigkeit vor dem Wechsel in den Ruhestand ist häufiger und länger andauernd geworden und mehr ältere Beschäftigte nehmen Altersteilzeit und nutzen diese für einen vorzeitigen $\mathrm{Ab}$ schied. Die geblockte Altersteilzeit hat - zusammen mit der Altersarbeitslosigkeit - den klassischen Vorruhestand ersetzt. 
Abbildung 4-5: Situation der 66- bis 71-Jährigen vor Beginn der Altersrente, nach Bildung, im Jahr 2014 (in Prozent)

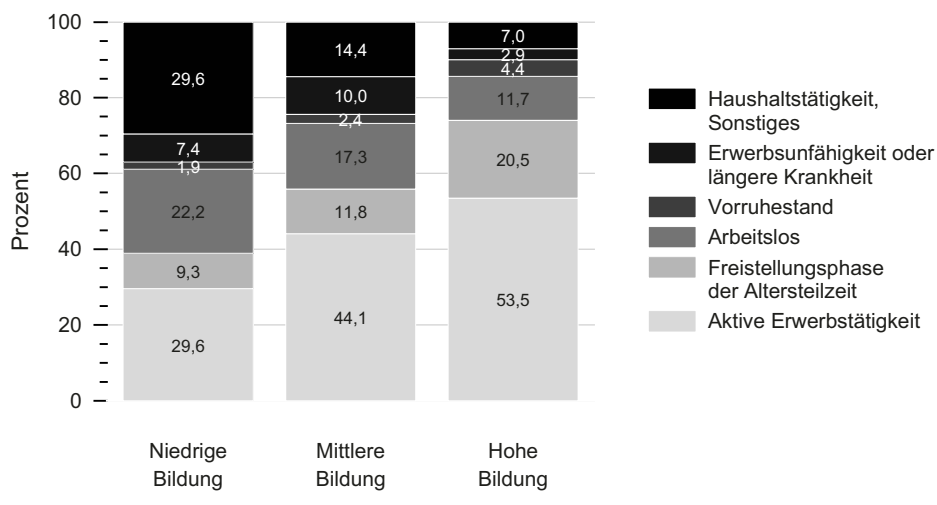

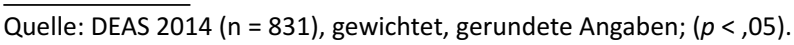

Signifikante Bildungsunterschiede bei allen Kategorien außer bei Erwerbsunfähigkeit, längerer Krankheit und Haushaltstätigkeit, Sonstiges.

\subsection{Verbreitung der Altersteilzeit}

Im DEAS werden seit dem Jahr 2002 alle abhängig Beschäftigten ab 55 Jahren gefragt, ob sie eine Altersteilzeitbeschäftigung ausüben. Wenn sie dies nicht machen (oder noch jünger als 55 Jahre sind), sollen sie angeben ob sie beabsichtigten, vor dem Ruhestand in die Altersteilzeit zu gehen. Wie die Ergebnisse für die älteren Beschäftigten ab 55 Jahren zeigen, ist es zwischen 2002 und 2008 zu einer deutlichen Zunahme der ausgeübten oder geplanten Altersteilzeit gekommen. Im Jahr 2008 befanden sich 16,9 Prozent der 55- bis 65-jährigen Beschäftigten in der Altersteilzeit, weitere 15,4 Prozent beabsichtigten eine Inanspruchnahme (Abbildung 4-6).

\section{Nach Abschaffung der staatlichen Förderung sinkt die Inanspruchnahme von Altersteilzeit wieder.}

Dieser Trend hat sich allerdings nicht fortgesetzt. Denn sechs Jahre später, im Jahr 2014, befinden sich nur noch 6,9 Prozent der über 55-jährigen Beschäftigten in der Altersteilzeit und nur noch 11,5 Prozent rechnen damit, sie in Anspruch zu nehmen. Dieser starke Rückgang stützt die Vermutung, dass das Auslaufen der staatlichen Förderung der Arbeitgeber dazu geführt hat, dass weniger ältere Beschäftigte die Möglichkeit zur Altersteilzeit erhalten bzw. damit rechnen. 


\section{Abbildung 4-6: Praktizierte und geplante Altersteilzeit der abhängig Beschäftigten im Alter von 55 bis 65 Jahren, in den Jahren 2002, 2008 und 2014 (in Prozent)}

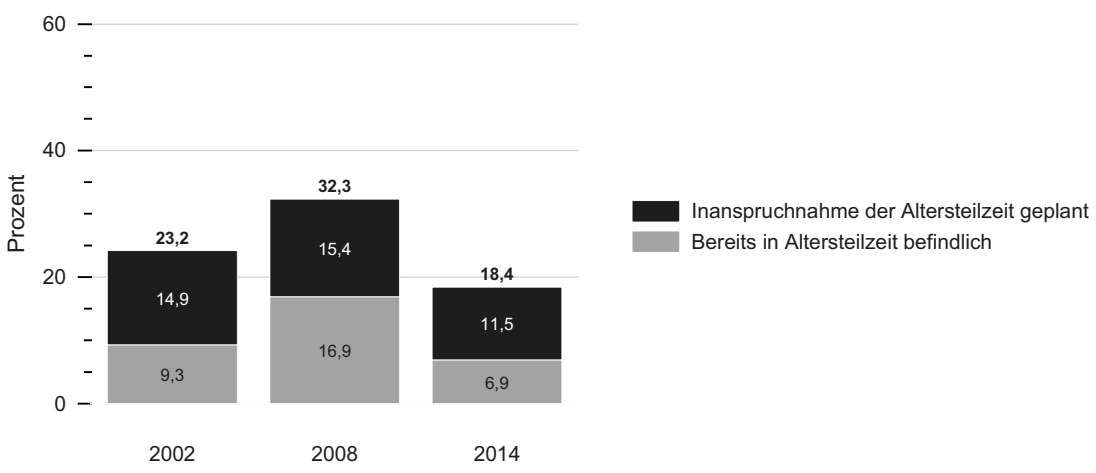

Quelle: DEAS $2002(n=221), 2008(n=609), 2014(n=838)$, gewichtet, gerundete Angaben; $(p<, 05)$. Unterschiede zwischen 2002 und 2008 sowie zwischen 2008 und 2014 für alle Kategorien signifikant.

Ein genauerer Blick auf die aktuelle Ausübung oder Planung von Altersteilzeit zeigt, dass deren Inanspruchnahme in hohem Maße mit dem Bildungsniveau der Beschäftigten, dem Wirtschaftszweig und der Größe des Betriebs assoziiert ist. Vergleichsweise geringe Quoten der praktizierten und geplanten Inanspruchnahme haben ältere Beschäftigte mit niedriger Bildung (ohne abgeschlossene Berufsausbildung) sowie Beschäftigte in kleinen Betrieben und außerhalb des Industriesektors (Abbildung 4-7). Eine relativ hohe Inanspruchnahme findet sich bei Beschäftigten mit mittlerer und höherer Bildung (abgeschlossene Berufsausbildung, Aufstiegsfortbildung oder Studienabschluss) und in Industrie- und Großbetrieben. Zudem kommen Männer und Beschäftigte in den alten Bundesländern etwas häufiger als Frauen und Beschäftigte in den neuen Bundesländern in den Genuss von Altersteilzeit; diese Unterschiede sind jedoch statistisch nicht signifikant. Zusammengenommen nutzen demnach überdurchschnittlich oft qualifizierte Beschäftigte in großen Industriebetrieben die Altersteilzeit als einen finanziell gut abgesicherten Übergangspfad in den Ruhestand.

Dabei wird die Altersteilzeit eher selten dafür verwendet, durch eine reduzierte Wochenarbeitszeit länger am Arbeitsprozess teilzuhaben und einen gleitenden Übergang in den Ruhestand vollziehen zu können. Im Jahr 2014 plant oder praktiziert mit 76,1 Prozent immer noch die große Mehrheit das Blockmodell, das einen frühzeitigen Abschied aus der aktiven Berufstätigkeit durch den Wechsel in die Freistellungsphase ermöglicht. Dennoch ist eine allmähliche Zunahme des Interesses am Weiterarbeiten mit halber Stundenzahl bis zum Rentenbeginn zu beobachten. Der Anteil des echten Teilzeitmodells an allen praktizierten und beabsichtigten Regelungen hat sich zwischen 2002 und 2014 von 13,6 auf 21,0 Prozent erhöht (ohne Abbildung). Im Jahr 2014 sind 2,9 Prozent noch unentschieden, welches Modell sie ausüben möchten.

Diejenigen, die in Altersteilzeit mit reduzierter Wochenstundenzahl bis zum Rentenbeginn berufstätig sind oder bleiben möchten, planen mit durchschnittlich 63,2 Jahren ihre Berufstätigkeit zu beenden. Dies ist rund ein Jahr später als bei älteren Arbeitskräften, die das Blockmodell der Altersteilzeit praktizieren oder beabsichtigen (62,3 Jahre). Einen noch späteren Ausstieg aus dem Erwerbsleben planen allerdings mit durchschnittlich 63,8 Jahren die über 55-Jährigen, die gar keine Altersteilzeit praktizieren oder beabsichtigen. In der bisherigen Form und bei den gegenwärtigen Anwendern trägt die Altersteilzeit daher offensichtlich noch nicht zu einem längeren Verbleib im Erwerbsleben bei.

Wie wird es weitergehen? Wird das Ausstiegsalter weiter steigen? Hinweise darauf las- 
sen sich aus den Plänen der heute und zukünftig älteren Beschäftigten gewinnen. Sie geben Aus- kunft, inwieweit die Betroffenen sich darauf einstellen, länger erwerbstätig zu bleiben.

\section{Abbildung 4-7: Praktizierte oder geplante Altersteilzeit der 55- bis 65-jährigen Beschäftigten nach verschiedenen Merkmalen, im Jahr 2014 (in Prozent)}

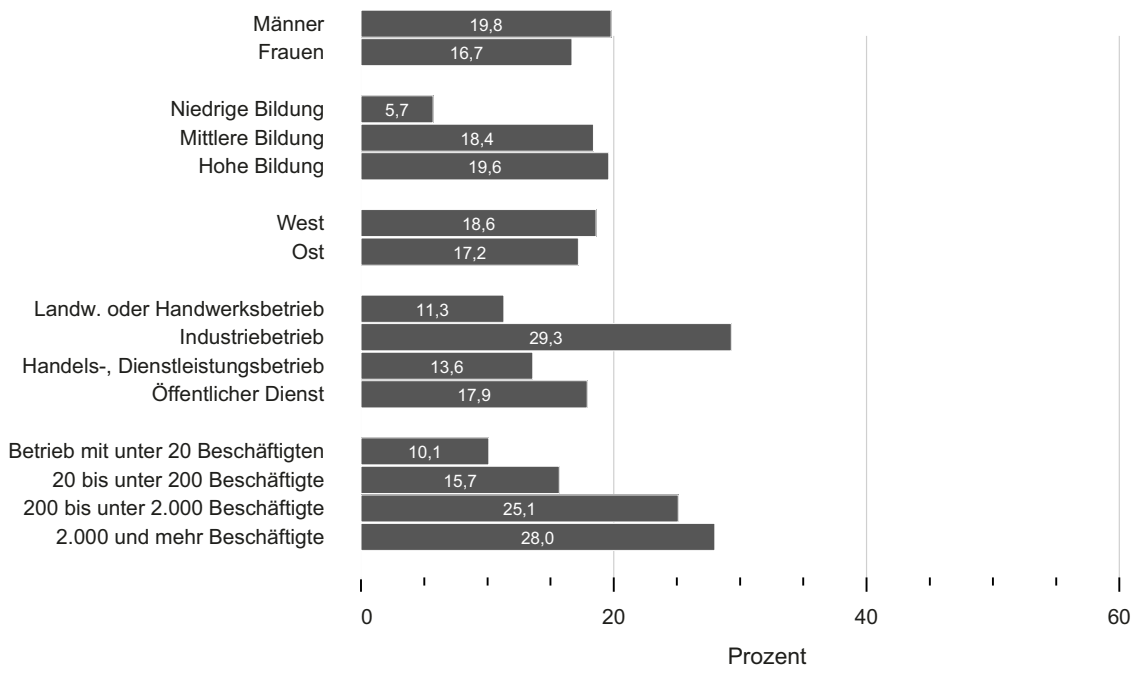

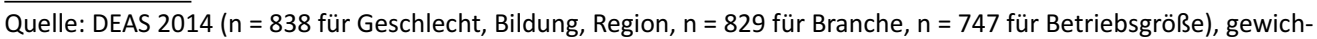
tet, gerundete Angaben; $(p<, 05)$.

Unterschied zwischen niedrigster und höchster Bildungsgruppe signifikant. Anteil in Industriebetrieben signifikant höher als in anderen Branchen. Anteil in Betrieben mit unter 200 Beschäftigten signifikant niedriger als in Betrieben ab 200 Beschäftigten. Geschlechts- und Regionsunterschied nicht signifikant.

Dabei wird die Altersteilzeit eher selten dafür verwendet, durch eine reduzierte Wochenarbeitszeit länger am Arbeitsprozess teilzuhaben und einen gleitenden Übergang in den Ruhestand vollziehen zu können. Im Jahr 2014 plant oder praktiziert mit 76,1 Prozent immer noch die große Mehrheit das Blockmodell, das einen frühzeitigen Abschied aus der aktiven Berufstätigkeit durch den Wechsel in die Freistellungsphase ermöglicht. Dennoch ist eine allmähliche Zunahme des Interesses am Weiterarbeiten mit halber Stundenzahl bis zum Rentenbeginn zu beobachten. Der Anteil des echten Teilzeitmodells an allen praktizierten und beabsichtigten Regelungen hat sich zwischen 2002 und 2014 von 13,6 auf 21,0 Prozent erhöht (ohne Abbildung). Im Jahr 2014 sind 2,9 Prozent noch unentschieden, welches Modell sie ausüben möchten.

Diejenigen, die in Altersteilzeit mit reduzierter Wochenstundenzahl bis zum Renten- beginn berufstätig sind oder bleiben möchten, planen mit durchschnittlich 63,2 Jahren ihre Berufstätigkeit zu beenden. Dies ist rund ein Jahr später als bei älteren Arbeitskräften, die das Blockmodell der Altersteilzeit praktizieren oder beabsichtigen (62,3 Jahre). Einen noch späteren Ausstieg aus dem Erwerbsleben planen allerdings mit durchschnittlich 63,8 Jahren die über 55-Jährigen, die gar keine Altersteilzeit praktizieren oder beabsichtigen. In der bisherigen Form und bei den gegenwärtigen Anwendern trägt die Altersteilzeit daher offensichtlich noch nicht zu einem längeren Verbleib im Erwerbsleben bei.

Wie wird es weitergehen? Wird das Ausstiegsalter weiter steigen? Hinweise darauf lassen sich aus den Plänen der heute und zukünftig älteren Beschäftigten gewinnen. Sie geben Auskunft, inwieweit die Betroffenen sich darauf einstellen, länger erwerbstätig zu bleiben. 


\subsection{Ausstiegspläne der Erwerbstätigen}

In jeder Erhebungswelle des DEAS werden die noch nicht im Ruhestand befindlichen Erwerbstätigen (Personen ohne Bezug einer eigenen Altersrente oder Pension) gefragt, in welchem Alter sie planen, ihre Erwerbstätigkeit zu beenden. Abbildung 4-8 enthält die zu Kategorien zusammengefassten Antworten der unter 60-Jährigen. Es wird deutlich, dass ein immer kleiner werdender Anteil einen Frühausstieg mit 60 Jahren oder jünger plant. Anfangs war die zunehmende Abkehr vom Plan eines frühen Ruhestands noch nicht verbunden mit konkreten neuen Altersvorstellungen für den eigenen Abschied. Dies zeigt sich in der hohen Quote der Kategorie „weiß noch nicht“ im Jahr 2002. In den darauffolgenden Jahren ist die Ungewissheit konkreten Vorstellungen zum Erwerbsausstieg in einem höheren Alter gewichen. Zugenommen hat sowohl der Anteil derer die planen, mit 61 bis 64 Jahren aufzuhören, als auch derjenigen die davon ausgehen, erst mit 65 Jahren oder später ihre Erwerbstätigkeit zu beenden. Mittlerweile beabsichtigt ein Fünftel der 40- bis 59-Jährigen, mit 65 Jahren in den Ruhestand zu gehen und ein weiteres Fünftel plant einen noch späteren Erwerbsausstieg (Abbildung 4-8).

\section{Immer mehr Erwerbstätige planen, bis zum Alter von 65 Jahren oder länger zu arbeiten.}

Der in der Politik und dem öffentlichen Diskurs propagierte und durch Reformmaßnahmen verfolgte Paradigmenwechsel von der Frühverrentung zum längeren Verbleib im Erwerbsleben findet ganz offensichtlich auch seinen Niederschlag in den Ausstiegsplänen der Erwerbstätigen. Dennoch darf nicht übersehen werden, dass immer noch mehr als 40 Prozent der 40- bis 59-Jährigen für sich persönlich einen Erwerbsausstieg vor Vollendung des 65. Lebensjahrs planen. Nicht alle wollen und können so lange erwerbstätig bleiben.

Abbildung 4-8: Geplantes Ausstiegsalter der 40- bis 59-jährigen Erwerbstätigen, in den Jahren 1996, 2002, 2008 und 2014 (in Prozent)

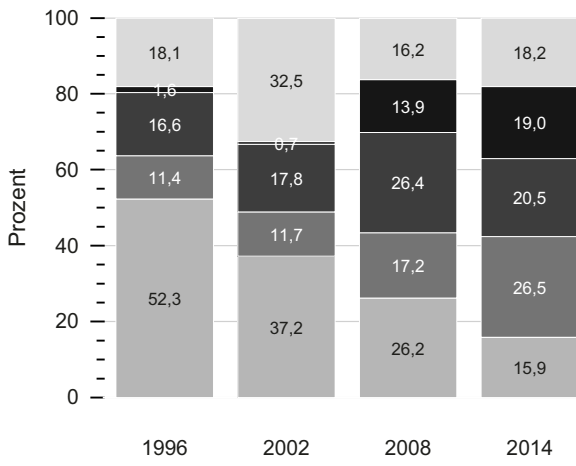

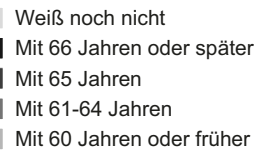

Quelle: DEAS $1996(n=1.756), 2002(n=1.019), 2008(n=2.187), 2014(n=2.119)$, gewichtet, gerundete Angaben; $(p<, 05)$.

Signifikante Veränderung aller Kategorien über die Erhebungsjahre. Anteil der Kategorie ,weiß noch nicht' im Jahr 2002 signifikant höher als in den anderen Jahren.

So gibt es deutliche Geschlechts- und Bildungsunterschiede im geplanten Ausstiegsalter (Abbildung 4-9). Männer beabsichtigen häufiger als
Frauen, erst mit 65 Jahren oder später aus dem Beruf auszusteigen. Überdurchschnittlich häufig planen Personen mit höherem beruflichem Bil- 
dungsabschluss, hochqualifizierte Angestellte, solche mit Vorgesetztenfunktion sowie Beamte und Beamtinnen und Selbstständige einen späten Ausstieg. Bei den Verbeamteten dürfte die Sicherheit ihres Arbeitsplatzes, bei Selbstständigen die freien Entscheidungsmöglichkeiten solche Pläne begünstigen. Etwas überraschend planen Personen mit Facharbeiter- und Meisterstatus (neun von zehn davon sind Männer) vergleichsweise selten, bis zum Alter von 65 Jahren zu arbeiten. Dies kann damit zusammenhängen, dass diese häufiger in Großbetrieben beschäftigt sind, deren Belegschaft leichter in den Genuss von Angeboten zum vorzeitigen Ausstieg über Vorruhestandsregelungen und geblockter Altersteilzeit kommt (siehe Abschnitt 4.4). Da diese Beschäftigtengruppe zum Befragungszeitpunkt im Durchschnitt zudem die meisten Erwerbsjahre aufweist (31 Jahre), kalkulieren Facharbeiter und Meister möglicherweise auch häufiger mit der Option, frühzeitig mit oder ohne Abschläge in die Altersrente für langjährig oder besonders langjährig Versicherte (nach 35 oder 45 Versicherungsjahren) zu gehen.

Die Ausstiegspläne unterscheiden sich auch nach der Arbeitsbelastung der Tätigkeit. Im DEAS geben die Erwerbstätigen Auskunft dazu, ob und gegebenenfalls wie sehr sie sich belastet fühlen durch körperlich anstrengende oder einseitige Tätigkeiten, erschwerte Umweltbedingungen am Arbeitsplatz (Lärm, Hitze, Staub etc.), Stress oder viele neue Anforderungen (siehe Abschnitt 4.2). Beschäftigte, die sich in mindestens einem dieser Bereiche ziemlich oder sehr belastet fühlen, planen deutlich seltener, bis zum Alter von 65 Jahren erwerbstätig zu bleiben als Beschäftigte, die sich durch keinen dieser Aspekte hoch belastet fühlen. Belastende Arbeitsbedingungen verringern die Neigung länger erwerbstätig zu bleiben beziehungsweise die subjektive Erwartung, die Tätigkeit bis zum 65. Lebensjahr ausüben zu können.

\section{Abbildung 4-9: Geplanter Ausstieg mit 65 und mehr Jahren der 40- bis 59-jährigen Erwerbstätigen nach verschiedenen Merkmalen, im Jahr 2014 (in Prozent)}

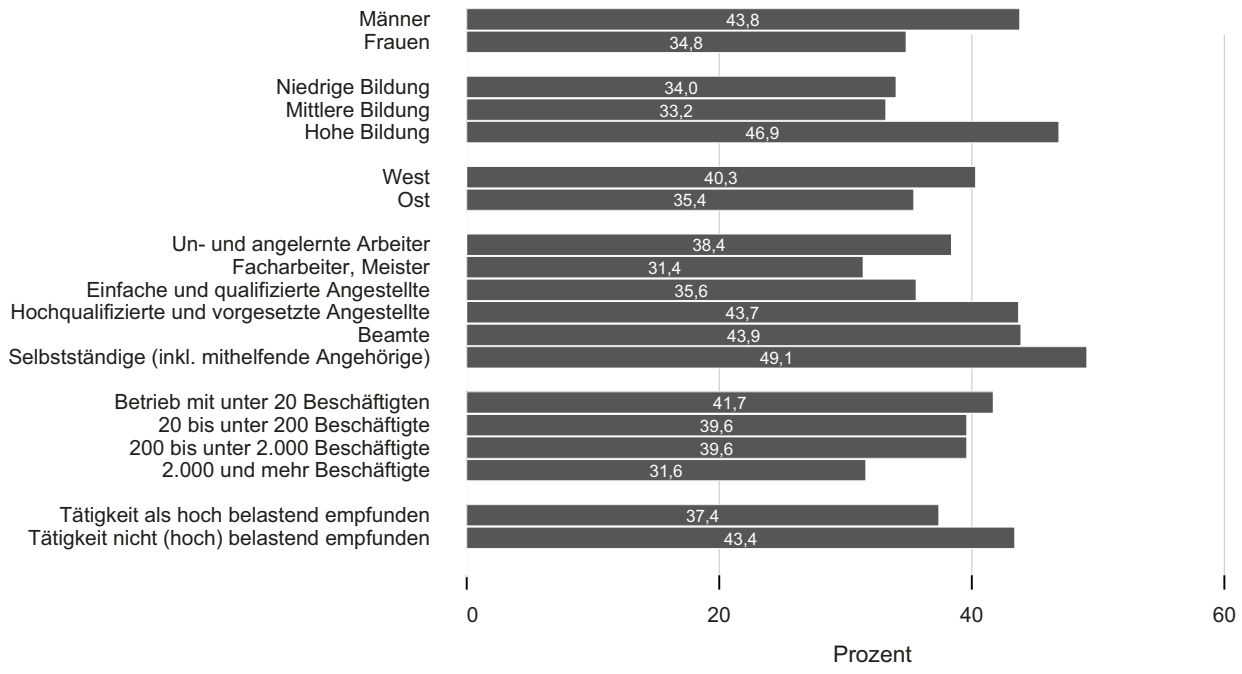

Quelle: DEAS 2014 ( $n=2.119$ für Geschlecht, Bildung, Region, Arbeitsbelastung, $n=2.105$ für berufliche Stellung, $\mathrm{n}=1.936$ für Betriebsgröße), gewichtet, gerundete Angaben; $(p<, 05)$.

Signifkante Unterschiede zwischen Geschlechtern und nach Arbeitsbelastung. Signifikant höherer Anteil bei Hochgebildeten im Vergleich zu anderen Bildungsgruppen. Signifikant niedrigerer Anteil bei Facharbeitern, Meistern im Vergleich zu jeder anderen beruflichen Stellung und in Betrieben mit 2000 und mehr Beschäftigten im Vergleich zu jeder anderen Betriebsgrößenkategorie. 
Insgesamt gibt es bei den noch im Erwerbsleben Stehenden zwar einen deutlichen Trend zur geplanten längeren Ausübung der Berufstätigkeit, allerdings begleitet von markanten Unterschieden nach beruflichen und betrieblichen Merk- malen. Dies lässt erwarten, dass das tatsächliche Übergangsalter zwar weiter ansteigen, es aber nicht allen möglich sein wird, bis zum Erreichen der Regelaltersgrenze durchzuarbeiten.

\subsection{Diskussion und Implikationen}

Die aufgezeigte Entwicklung des Übergangsalters in den Ruhestand entspricht insgesamt der von Politik und Wirtschaft propagierten Richtung des längeren Verbleibs im Erwerbsleben und des späteren Rentenbeginns. Der in den Retrospektivangaben des DEAS erkennbare Anstieg des Rentenalters ab den Anfang der 19040er Jahre Geborenen zeigt sich auch in den Zahlen der Rentenversicherung (Deutsche Rentenversicherung 2015). Allerdings hat es etwas gedauert, bis nach dem Renteneintrittsalter auch das Erwerbsaustrittsalter zu steigen begann. Noch bis Ende des letzten Jahrzehnts vergrößerte sich die zeitliche Lücke nach der Aufgabe der Berufstätigkeit bis zum Rentenbeginn, verringert sich aber seit einigen Jahren wieder. Dennoch hat bei den betrachteten Kohorten der Anteil derer abgenommen, die bis unmittelbar vor dem Renteneintritt erwerbstätig waren. Ein wachsender Anteil älterer Arbeitskräfte wurde vor dem Rentenbeginn arbeitslos und die Dauer der Arbeitslosigkeit bis zum Wechsel in die Altersrente hat sich verlängert. Besonders betroffen davon waren ostdeutsche Frauen, bei denen es im Beobachtungszeitraum zwischen 1996 und 2014 zu einem massiven Umbruch der Übergangspfade in den Ruhestand weg vom Arbeiten bis zur Rente hin zur mehrjährigen Arbeitslosigkeit vor dem Renteneintritt kam. Auch Beschäftigte mit niedrigerem Bildungs- und Qualifikationsniveau beenden ihr Erwerbsleben vergleichsweise häufig mit Arbeitslosigkeit. Personen mit hohem Bildungsniveau gelingt es hingegen überdurchschnittlich oft, entweder bis zum Rentenbeginn aktiv erwerbstätig zu bleiben oder aus der gut abgesicherten Position der Altersteilzeit in den Ruhestand wechseln. Ähnliche Befunde zum Wandel und der sozialen Aus- differenzierung der Übergangspfade und des Übergangsalters in den Ruhestand zeigen sich auch in anderen Kohortenstudien (vgl. Rasner \& Etgeton 2014; Buchholz, Rinklake, \& Blossfeld 2014; Schröber, Micheel, \& Cihlar 2015; Brussig 2015). Die Ergebnisse sind noch weitgehend unbeeinflusst von der neu geschaffenen Möglichkeit des vorgezogenen Rentenzugangs ohne Abschläge in die Altersrente für besonders langjährig Versicherte. Nach Informationen der Rentenversicherung haben davon im Jahr 2014 mehr als 150.000 Personen Gebrauch gemacht (Deutsche Rentenversicherung 2015). Es ist davon auszugehen, dass diese Rentenzugangsoption einerseits die Zahl der direkten - jedoch vorzeitigen - Ruhestandsübergänge aus der Erwerbstätigkeit erhöhen wird, andererseits die sozialen und Geschlechtsunterschiede in den Übergangspfaden verstärkt. Denn die Zugangsvoraussetzungen von mindestens 45 Beitragsjahren erfüllen in erster Linie Beschäftigtengruppen ohne längere Erwerbsunterbrechungen aufgrund von Arbeitslosigkeit oder Sorgetätigkeiten in der Familie.

Eine weitere Reformmaßnahme, die die Optionen des Übergangs in den Ruhestand tangiert, war die Abschaffung der Förderung der Altersteilzeitbeschäftigung durch die Bundesagentur für Arbeit. Dies führte zur Frage, ob dies einen Rückgang der Inanspruchnahme von Altersteilzeit durch ältere Beschäftigte zur Folge hat. Die Befunde zeigen deutlich, dass der Trend gestoppt wurde, vor dem Ruhestand in die Altersteilzeitbeschäftigung zu wechseln. Denn die tatsächliche Inanspruchnahme bei den 55- bis 65-jährigen abhängig Beschäftigten hat sich - nach vorheriger Zunahme - in den sechs Jahren zwischen 2008 und 2014 mehr als halbiert; auch der Anteil derer, 
die eine Altersteilzeit beabsichtigen, ging zurück. Offenbar hat die Abschaffung der staatlichen Förderung zu einer Verringerung des Angebots und der Nachfrage nach Altersteilzeit geführt.

Es zeigt sich außerdem, dass insbesondere qualifizierte Beschäftigte in Großbetrieben des industriellen Sektors in den Genuss der Altersteilzeit kommen. Die Mehrzahl der älteren Arbeitskräfte, die eine Altersteilzeitbeschäftigung ausüben oder beabsichtigen, wählt das Blockmodell, das ihnen einen frühzeitigen Abschied aus der aktiven Berufstätigkeit ermöglicht. Zwar hat das Gleichverteilungsmodell der Altersteilzeit, das ein Weiterarbeiten mit halber Stundenzahl bis zum Rentenbeginn vorsieht, an Bedeutung gewonnen, mehr als drei Viertel praktizieren oder planen jedoch immer noch das Blockmodell. Große Betriebe haben diese Beschäftigungsform in der Vergangenheit häufig als eine kostengünstige Möglichkeit zur Personalanpassung durch frühzeitige Ausgliederung genutzt. Es bleibt abzuwarten, ob der Rückgang der Altersteilzeitangebote in Zukunft zu einem längeren Weiterarbeiten älterer Arbeitskräfte oder $\mathrm{zu}$ anderen Formen des vorzeitigen Ausstiegs führt, zum Beispiel über Arbeitslosigkeit, längere Krankheit oder Erwerbsminderung.

In welchem Maße sich die Entwicklung zu einem längeren Verbleib im Erwerbsleben fortsetzen wird, hängt auch von den Wünschen und Plänen der Beschäftigten ab. Inwieweit stellen sie sich darauf ein, länger erwerbstätig zu bleiben? $\mathrm{Zu}$ diesem Zweck wurde die Entwicklung des geplanten Ausstiegsalters der Erwerbstätigen untersucht. Insgesamt lässt sich ein deutlicher Trend zur geplanten längeren Ausübung der Erwerbsarbeit feststellen, allerdings begleitet von Unterschieden nach beruflichen und betrieblichen Merkmalen. Nicht alle Arbeitskräfte wollen und können bis zum Alter von 65 Jah- ren oder darüber hinaus erwerbstätig bleiben. Beispielsweise glaubt nur eine Minderheit derjenigen, die ihre Tätigkeit als hoch belastend empfinden, bis zur Regelaltersgrenze im Arbeitsprozess zu bleiben.

Der Abbau von Frühverrentungsmöglichkeiten hat zwar insgesamt dazu beigetragen, dass mehr ältere Arbeitskräfte länger berufstätig bleiben und die Abkehr von der Frühverrentungsmentalität forciert. Für diejenigen, die es jedoch nicht schaffen, bis zur Regelaltersgrenze zu arbeiten, besteht allerdings die Gefahr, am Ende ihres Arbeitslebens jahrelang arbeitslos $\mathrm{zu}$ sein und dann vorzeitig mit hohen Abschlägen verrentet zu werden. In den vergangenen Jahren zeigte sich bereits eine Zunahme der Altersarbeitslosigkeit. Eine weiter steigende Altersarbeitslosigkeit und eine sich verlängernde Dauer der Arbeitslosigkeit vor dem Übergang in den Ruhestand wären problematisch. Denn während der Arbeitslosigkeit werden im Allgemeinen nur noch geringe oder gar keine Rentenanwartschaften mehr erworben und der oft vorzeitige Rentenbeginn führt zu lebenslangen Abschlägen bei der Rentenhöhe. Dies erhöht das Risiko der Altersarmut. Die Tarifparteien und die Politik sind gefordert, Lösungen für eine Flexibilisierung des Übergangs in den Ruhestand zu finden. Einerseits sollte denjenigen, die länger arbeiten können und möchten, es möglichst einfach gemacht werden, dies zu realisieren. Andererseits sollten denjenigen, die aus gesundheitlichen oder Arbeitsmarktgründen dazu nicht in der Lage sind, flexible Möglichkeiten des vorzeitigen Übergangs in den Ruhestand eröffnet werden. Diese sollten finanziell zumindest soweit abgesichert sein, dass das kaum vermeidbare vorzeitige Ausscheiden aus dem Erwerbsleben zu keinem dauerhaften Abrutschen in die Armutszone führt.

\section{Literatur}

Brussig, M. (2015). Alter beim Übergang aus sozialversicherungspflichtiger Beschäftigung ist gestiegen (Altersübergangs-Report Nr. 2015-01). Gelsenkirchen: Institut Arbeit und Technik.
Brussig, M., Knuth, M., \& Wojtkowski, S. (2009). Altersteilzeit: Zunehmend Beschäftigungsbrücke zum späteren Renteneintritt (Altersübergangs-Report Nr. 200902). Gelsenkirchen: Institut Arbeit und Technik. 
Buchholz, S., Rinklake, A., \& Blossfeld, H.-P. (2014). Reversing Early Retirement in Germany. A Longitudinal Analysis of the Effects of Recent Pension Reforms on the Timing of the Transition to Retirement and on Pension Incomes. Comparative population studies, 38(4), 881-906.

CDU, CSU, \& SPD (2013). Deutschlands Zukunft gestalten. Koalitionsvertrag zwischen CDU, CSU und SPD, 18. Legislaturperiode. Berlin.

Deutsche Rentenversicherung (2015). Rentenversicherung in Zeitreihen. Berlin: Deutsche Rentenversicherung.

Engstler, H. (2006). Erwerbsbeteiligung in der zweiten Lebenshälfte und der Übergang in den Ruhestand. In: C. Tesch-Römer, H. Engstler \& S. Wurm (Hrsg.) Altwerden in Deutschland. Sozialer Wandel und individuelle Entwicklung in der zweiten Lebenshälfte (S. 85-154). Wiesbaden: VS Verlag für Sozialwissenschaften.

Fröhler, N., Fehmel, T., \& Klammer, U. (2013). Flexibel in die Rente. Gesetzliche, tarifliche und betriebliche Perspektiven. Berlin: edition sigma.

Naumann, D., \& Romeu Gordo, L. (2010). Gesellschaftliche Partizipation: Erwerbstätigkeit, Ehrenamt und Bil- dung. In: A. Motel-Klingebiel, S. Wurm \& C. Tesch-Römer (Hrsg.) Altern im Wandel. Befunde des Deutschen Alterssurveys (DEAS) (S. 118-141). Stuttgart: Kohlhammer.

Rasner, A., \& Etgeton, S. (2014). Rentenübergangspfade: Reformen haben großen Einfluss. DIW Wochenbericht, 81(19), 431-441.

Schröber, J., Micheel, F., \& Cihlar, V. (2015). Übergangskonstellationen in die Altersrente - Welche Rolle spielen Humankapital und betrieblicher Kontext? In: N. F. Schneider, A. Mergenthaler, U. M. Staudinger \& I. Sackreuther (Hrsg.) Mittendrin? Lebenspläne und Potenziale älterer Menschen beim Übergang in den Ruhestand (S. 195-216). Opladen/Berlin/Toronto: Budrich.

Simonson, J., Romeo Gordo, L., \& Titova, N. (2011). Changing employment patterns of women in Germany: How do baby boomers differ from older cohorts? A comparison using sequence analysis. Advances in Life Course Research, 16(2), 65-82.

Wanger, S. (2009). Altersteilzeit: Beliebt, aber nicht zukunftsgerecht. Nürnberg: Institut für Arbeitsmarktund Berufsforschung.

Open Access Dieses Kapitel wird unter der Creative Commons Namensnennung 2.5 International Lizenz (http://creativecommons.org/licenses/by/2.5/deed.de) veröffentlicht, welche die Nutzung, Vervielfältigung, Bearbeitung, Verbreitung und Wiedergabe in jeglichem Medium und Format erlaubt, sofern Sie den/die ursprünglichen Autor(en) und die Quelle ordnungsgemäß nennen, einen Link zur Creative Commons Lizenz beifügen und angeben, ob Änderungen vorgenommen wurden.

Die in diesem Kapitel enthaltenen Bilder und sonstiges Drittmaterial unterliegen ebenfalls der genannten Creative Commons Lizenz, sofern sich aus der Abbildungslegende nichts anderes ergibt. Sofern das betreffende Material nicht unter der genannten Creative Commons Lizenz steht und die betreffende Handlung nicht nach gesetzlichen Vorschriften erlaubt ist, ist für die oben aufgeführten Weiterverwendungen des Materials die Einwilligung des jeweiligen Rechteinhabers einzuholen. 